be informative in tracking changes in functional status, and identifying individuals at a high risk of falling to allow for preventative measures. We have developed a technology, called AMBIENT, which enables the frequent, accurate, unobtrusive, and cost-effective measurement of gait and balance parameters. The objective of this study was to demonstrate the feasibility of using AMBIENT for frequent assessment of mobility in people with dementia in a residential facility. Methods: We conducted a pilot longitudinal study with 20 participants (age: $76.9 \pm 6.7$ years, female: $50 \%$ ) in the geriatric psychiatry unit at the Toronto Rehabilitation Institute, an eighteen-bed inpatient dementia care unit for older adults with behavioral symptoms. The AMBIENT setup included radio frequency identification to identify study participants and a Microsoft Kinect sensor to track body posture. The system automatically monitored participants' gait as they walked within the view of the sensor during their daily routine and computed the spatiotemporal parameters of gait. Demographic and baseline descriptive measures were collected and falls events tracked. Results: On average, 97 walking sequences per person were collected over a length of stay of $46 \pm 37$ days. There were 14 falls among study participants: 12 participants did not fall during their length of stay, 4 fell once, 2 fell twice, and 2 fell 3 times. Quantitative measures of gait were stride length $(0.8 \pm 0.1 \mathrm{~m})$, stride time $(1.4 \pm 0.2 \mathrm{~s})$, cadence $(89.3 \pm 18.1 \mathrm{steps} / \mathrm{min})$, velocity $(0.6 \pm 0.1 \mathrm{~m} / \mathrm{s})$, step length asymmetry $(1.2 \pm 0.6)$, and step time asymmetry $(1.2 \pm 0.5)$. Conclusions: This pilot study demonstrates the feasibility of longitudinal tracking of gait over time in a residential dementia setting. Our long-term goal is to translate longitudinal gait parameters onto fall-risk measures. Machine learning techniques will be used to build a robust, multivariate predictive model capable of detecting changes in mobility and falls risk.

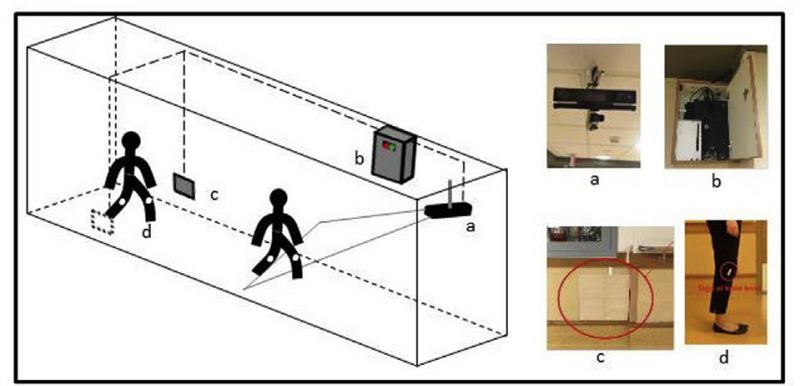

AMBIENT set-up in the hallway of Geriatric Psychiatry unit at the Toronto Rehabilitation Institute. AMBIENT set-up includes (a) a Kinect sensor, (b) a control box, and (c) two RFID antennas, (d) RFID tags were attached to participants' clothing at knee level.

\section{P1-555 IMPACT OF PATIENT MILD COGNITIVE IMPAIRMENT ON PHYSICIAN DECISION- MAKING FOR TREATMENT}

Emilie Blair ${ }^{1}$, Darin Zahuranec ${ }^{1}$, Kenneth M. Langa ${ }^{1}$, Jane Forman ${ }^{1,2}$, Bailey K. Reale ${ }^{1}$, Colleen Kollman ${ }^{3}$, Bruno Giordani ${ }^{4}$, Deborah Levine ${ }^{1},{ }^{1}$ University of Michigan, Ann Arbor, MI, USA; ${ }^{2}$ VA Ann Arbor Healthcare System, Ann Arbor, MI, USA; ${ }^{3}$ Kollman Research Services, Ann Arbor, MI, USA; ${ }^{4}$ Michigan Alzheimer's Disease Center, Ann Arbor, MI, USA. Contacte-mail: emiliebl@med.umich.edu
Background:Mild cognitive impairment (MCI) is the most common cognitive disorder of the elderly, affecting up to 1 in 5 adults 65 or older, 5.4 million Americans. Evidence suggests that older patients with MCI may be undertreated for cardiovascular events that are common at this age. In order to understand the reasons for this disparity, we explored the influence of a patient's MCI diagnosis on clinical decision making by physicians in a pilot study. Methods: Qualitative study using in-person, in-depth, semi-structured interviews and standard interview guide. Physicians $(n=18)$ from cardiology, neurology, and internal medicine at 1 academic medical center were recruited from a convenience sample of 39 physicians. We sought to understand whether and how a patient's having preexisting MCI has influenced physicians' decisions about 5 kinds of treatments or tests (surgery, invasive tests, non-invasive tests, rehabilitation, and preventive medication). We used qualitative content analysis to identify the unifying and recurrent themes. Results: Most participants (89\%) described MCI as influencing their recommendations for treatments and tests in at least one way. We identified five recurrent themes (Table 1). The majority of physicians $(61 \%)$ assumed that patients with MCI are older and frailer than patients with normal cognition. Half of the physicians believed that patients with MCI are likely to develop dementia and some treatments may increase dementia risk. Some physicians made assumptions that patients with MCI, compared to patients with normal cognition, do not understand treatment $(56 \%)$, are unable to comply with treatment (39\%), and want less treatment $(22 \%)$. Conclusions: MCI influences physician decision making for treatment. Without awareness of physician assumptions about MCI, a large portion of the elderly population may not get effective treatments.

\section{Table 1}

Themes of the Influence of Patient Mild Cognitive Impairment on Physician Decision Making for Treatment

Patients with MCI are older and frailer than patients with normal cognition.

- "If you look at studies where they show benefit for statins, it's predicated on having a certain number of years of life expectancy. And if you're past that, then there's not much data showing benefit for those medications."

- "I can imagine putting some weight into the fact that they have mild cognitive impairment in that situation, if I'm trying to decide whether or not to recommend the person undergo surgery or not."

- "MCI might be a variable that I could talk myself into being a non-perfect circumstance."

Patients with MCI are likely to develop dementia and treatment may increase dementia risk.

Patients with MCI want less medical treatment than patients with normal cognition.
- "I would tell them upfront that there is a risk of patients with MCI progressing into a condition with dementia"

- "I would be disinclined to sedate you, if you had to go through those tests"

- "And just as with communication, I imagine that navigating those decisions and those preference based things is going to be always harder in MCI. It's going to be probably harder for people to identify what their preferences are, to articulate them when they exist. And then to navigate these decisions once they happen." 